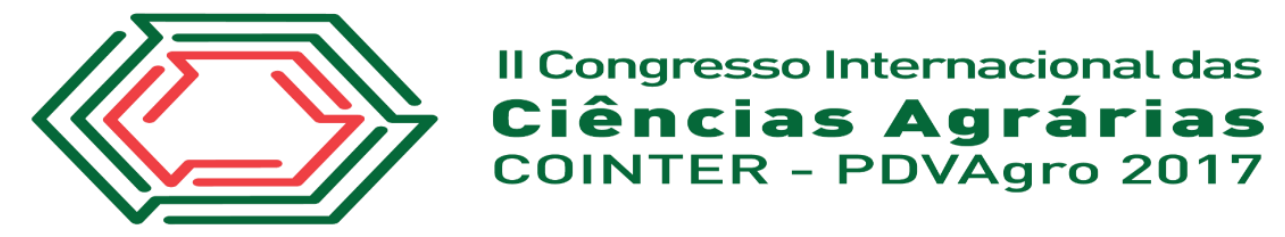

\title{
DIVERSIDADE E ABUNDÂNCIA DA FAUNA COLEOPTERA AÉREA EM PLANTIOS COMERCIAIS DE CÔCO (Cocos nucifera $\mathbf{L}$.)
}

Fernanda Valente Penner ${ }^{1}$; Natália Cristina de Almeida Azevedo ${ }^{2}$; Lucas Faro Bastos ${ }^{3}$; Paulo Manoel Lins ${ }^{4}$; Telma Fátima Vieira Batista ${ }^{5}$

\section{Introdução}

A importância dos insetos vai além de situações cotidianas como polinização e produção de mel. Todas as espécies de insetos são constituintes de um conjunto maior de vida, de tal modo que a sua perda ou aumento populacional desordenado possa vir a comprometer a diversidade e abundância de outros organismos dependentes deles. Para Gullan et al, (2017), alguns insetos podem ser considerados como "espécies-chave", visto que sua importância no agroecossistema é significativa onde a desordem poderia ocasionar desequilíbrios que afetam o meio ambiente como um todo.

A adoção de levantamentos fitossanitários como estudo inicial para as práticas de manejo sustentável é um dos principais focos para o conhecimento de um inseto-praga, visto que, é necessário compreender primeiramente a ecologia destes para então vir a estudar a situação ambiental do local em que se encontram.

Com base no exposto, objetivou-se determinar a diversidade e a abundância das famílias de coleópteros associados a plantios comerciais de coco, com ênfase as famílias de possíveis causadores de injúrias e predadores, propiciando subsídios futuros ao manejo integrado de pragas na cocoicultura paraense.

\section{Fundamentação Teórica}

O coqueiro é uma palmeira importante para a alimentação humana, geralmente adaptada a climas tropicais e subtropicais, preferencialmente com pluviosidade superior a $1.500 \mathrm{~mm}$. Possui como a mais provável origem as ilhas do sudeste asiático. É uma planta da família Arecaceae com crescente potencial produtivo. Pode emitir até 20 inflorescências produtivas por ano e cada uma

\footnotetext{
${ }^{1}$ Graduanda em Engenharia Florestal, Universidade Federal Rural da Amazônia, fernandavpenner@gmail.com

${ }^{2}$ Graduanda em Engenharia Florestal, Universidade Federal Rural da Amazônia, naty.azeved@gmail.com

${ }^{3}$ Mestrando em agronomia, Universidade Federal Rural da Amazônia, lucas.fbufra@gmail.com

${ }^{4}$ Doutor em ciências agrárias, gerência de pesquisas na empresa Sococo agroindústrias da Amazônia -Santa Isabel do Pará-Pa, paulom@sococo.com.br

${ }^{5}$ Doutora em ciências agrárias, Universidade Federal Rural da Amazônia, telma.batista@ufra.edu.br
} 
chega alcançar até 32 frutos/cacho (Aragão, 1998; Aragão, 1997). Entretanto, esse cultivo pode ser atacado por coleópteros. A ordem coleóptera é considerada uma das mais importantes entre os artrópodes, pois possui a maior representatividade da classe devido ao alto número de espécies conhecidas, que somam cerca de $40 \%$ do total de espécies descritas (Triplehorn \& Johnson, 2015). Características morfológicas que identificam essa ordem é a presença de dois pares de asas, sendo o primeiro do tipo élitro e segundo membranoso. Os indivíduos podem variar de tamanho, sendo encontrados em larga escala, que compreende espécimes de $200 \mathrm{~mm}$ de comprimento até extremamente diminutos, menores do que $1 \mathrm{~mm}$. (Gallo et al, 2002).

Muitas são as pragas que causam danos ao coqueiro, destacam-se os coleópteros Rhychophorus palmarum L. (broca-do-olho-do-coqueiro) e Rhinostomus barbirostris Fabri. (brocado-estipe) ambos da família Curculionidae, podem causar desde problemas facilmente controláveis até distúrbios letais, altamente prejudiciais a cultura (Gallo et al, 2002; Lemos et al, 2016).

\section{Metodologia}

O estudo foi realizado em área comercial da empresa Sococo, localizada no município de Santa Isabel do Pará-Pa. Segundo a classificação de Köppen-Geiger a região possui clima do tipo Afi, e é caracterizada por elevados índices de precipitação, com valores em torno de $3.000 \mathrm{~mm}$ (SEMA, 2017).

O levantamento faunístico foi executado em uma parcela de 8,08 ha, com a utilização de 40 armadilhas amarelas adesivas do tipo painel nas dimensões 23 x $28 \mathrm{~cm}$, sendo alocadas nas copas dos coqueiros com espaçamento de $70 \mathrm{~m}$ entre linhas de cultura e $21 \mathrm{~m}$ entre plantas, de forma que a sua distribuição sistemática estivesse de forma apta para obter a melhor representatividade da entomofauna aérea.

A coleopterofauna coletada foi triada, quantificada e identificada até família, através de chaves dicotômicas no Laboratório de Proteção de Plantas, pertencente a Universidade Federal Rural da Amazônia, no campus de Belém.

As análises estatísticas dos índices de dominância, abundância e frequência foram calculados utilizando o software ANAFAU (Silveira Neto, 1976; Moraes et al., 2003).

\section{Resultados e Discussões}

Foi observada grande diversidade biológica. Foram coletados 604 indivíduos pertencentes a 11 famílias. As famílias Coccinellidae e Curculionidae apresentaram maior frequência com 79,47\% e 6,62\%, dos indivíduos, seguidos de Bruchidae, Chrysomelidae, Meloidae, Scarabaeidae, 
Bostrichidae, Cerambycidae, Tenebrionidae, Carabidae e Cicindelidae que apresentaram 5,30\%, $4,14 \%, 0,99 \%, 0,83 \%, 0,83 \%, 0,50 \%, 0,50 \%, 0,33 \%$ e $0,17 \%$ respectivamente, $0,33 \%$ não foram identificados.

Tabela 1. Número de indivíduos e frequência (\%) de famílias coletadas em plantios comerciais de coco em Santa Isabel do Pará, 2017.

\begin{tabular}{lcc}
\hline \multicolumn{1}{c}{ Família } & $\mathbf{N}^{\mathbf{0}}$ de indivíduos & Frequência (\%) \\
\hline Bostrichidae & 5 & 0,83 \\
Bruchidae & 32 & 5,30 \\
Carabidae & 2 & 0,33 \\
Cerambycidae & 3 & 0,50 \\
Chrysomelidae & 25 & 4,14 \\
Cicindelidae & 1 & 0,17 \\
Coccinellidae & 480 & 79,47 \\
Curculionidae & 40 & 6,62 \\
Meloidae & 6 & 0,99 \\
Scarabaeidae & 5 & 0,83 \\
Tenebrionidae & 3 & 0,50 \\
N.I. & 2 & 0,33 \\
\hline Total de indivíduos & $\mathbf{6 0 4}$ & $\mathbf{1 0 0}$ \\
\hline
\end{tabular}

Segundo Lixa (2008) os predadores da família Coccinellidae, conhecidos popularmente como joaninhas, atuam como inimigos naturais das pragas agrícolas e exercem um papel importante no controle biológico de insetos e ácaros fitófagos. Como exemplo, tem-se o caso da joaninha Crytolaemus montrouzieri Mulsant, tendo sido enviada do Chile para o Brasil, com o intuito de controlar a Planococcus citri Risso, conhecida vulgarmente como cochonilha branca dos citros (GRAVENA, 2017).

$\mathrm{Na}$ família Curculionidae esta presente uma das principais pragas de coqueiros Rhynchophorus palmarum, conhecida como Broca do olho do coqueiro, quando em forma de larva se alimentam e destroem os tecidos meristemáticos, criando galerias em todas as direções, podendo ocasionar até a morte do vegetal (ALENCAR et al, 2002).

Em relação a analise das famílias encontradas, a família Coccinellidae foi considerada como super dominante, super abundante, super frequente e constante; seguida de Bruchidae, Chrysomelidae e Curculionidae as quais classificadas como dominantes, muito abundante e muito frequente, além de que, essas 5 famílias foram vistas como predominantes na área (Tabela 2). As demais famílias foram classificadas como não dominantes, comuns ou dispersas e frequentes ou pouco frequentes, não possuindo quantidades de indivíduos significantes para a análise. 
Tabela 2. Análise faunística das famílias de coleopteros associados a plantios comerciais de coco em Santa Isabel do Pará, Pa, 2017.

\begin{tabular}{|c|c|c|c|c|}
\hline FAMÍLIAS & DO & $\mathbf{A B}$ & FR & $\mathrm{CO}$ \\
\hline Bostrichidae & ND & $\mathrm{C}$ & $\mathrm{F}$ & W \\
\hline * Bruchidae & D & $\mathrm{MA}$ & MF & W \\
\hline Carabidae & ND & $\mathrm{Di}$ & $\mathrm{PF}$ & W \\
\hline Cerambycidae & ND & C & $\mathrm{F}$ & w \\
\hline * Chrysomelidae & D & $\mathrm{MA}$ & MF & W \\
\hline Cicindelidae & ND & $\mathrm{Di}$ & $\mathrm{PF}$ & W \\
\hline * Coccinellidae & SD & SA & SF & w \\
\hline * Curculionidae & D & MA & MF & w \\
\hline Meloidae & D & C & $\mathrm{F}$ & w \\
\hline Scarabaeidae & ND & C & $\mathrm{F}$ & W \\
\hline Tenebrionidae & ND & C & $\mathrm{F}$ & W \\
\hline N.I. & ND & $\mathrm{Di}$ & $\mathrm{PF}$ & w \\
\hline
\end{tabular}

Legenda: $(\mathrm{DO})=$ Dominância; $(\mathrm{ND})=$ não dominante; $(\mathrm{D})=$ dominante; $(\mathrm{SD})=$ super dominante; $\mathrm{AB})=$ Abundância; $(\mathrm{C})=$ comum; $(\mathrm{R})=$ raro; $(\mathrm{MA})=$ muito abundante; $(\mathrm{SA})=$ super abundante; $\mathrm{Di})=$ dispersa; $(\mathrm{FR})=$ Frequência; $(\mathrm{F})=$ frequente; $(\mathrm{PF})=$ pouco frequente; $(\mathrm{MF})=$ muito frequente; $(\mathrm{SF})=$ super frequente; $(\mathrm{CO})=$ Constância; $(\mathrm{W})=$ constante *famílias predominantes (bioindicadores).

Segundo Silveira Neto et al. (1976) os organismos dominantes possuem habilidades para impactos causados pelo meio e corresponde de forma sadia ou equilibrada, permanece no local, modifica o ambiente ao redor, e podendo intervir nas comunidades de organismos diferentes, e até causar o desaparecimento destes, como é o caso dos Coccinellideos que foi encontrado com frequência de 79,47\% nas armadilhas. As famílias Bostrichidae, Bruchidae, Cerambycidae, Curculionidae, Scarabaeidae, Meloidae e Tenebrionidae foram consideradas pragas, enquanto que, Chrysomelidae, Cicindelidae, Coccinellidae e Carabidae são predadores.

Tabela 3. Índices de diversidade de famílias de coleópteros coletados em plantios comerciais de coco em Santa Isabel do Pará, Pa, 2017

\begin{tabular}{lc}
\hline \multicolumn{1}{c}{ Índices de diversidade } & Resultados \\
\hline Índice de Shannon $\left(H^{\prime} \pm I C\right)$ & 1,7950 \\
Índice de Riqueza (Margalef) & 2.0746 \\
Índice de Equitabilidade & 0.7486
\end{tabular}

Médias ao nível de 5\% (p = 0,05), IC: Intervalo de confiança de $H^{\prime}$.

Os índices faunísticos demonstraram que: o Índice de Shannon utilizado na avaliação da diversidade de famílias na área, 1,7950 foi considerado baixo, entretanto, com alta riqueza de famílias que correspondeu ao Índice de Margalef, 2,0746, acima de 2 e alto Índice de Equitabilidade, o qual foi empregado para quantificar a uniformidade das famílias encontradas na área, tendo 
resultado de 0,7486 .

\section{Conclusões}

A fauna coleóptera associada a plantios comerciais de coqueiro no Pará é diversificada. Apresenta 7 famílias como Bostrichidae, Bruchidae, Cerambycidae, Curculionidae, Scarabaeidae, Meloidae e Tenebrionidae como potenciais pragas ao cultivo e apenas 4 famílias como Chrysomelidae, Cicindelidae, Coccinellidae e Carabidae consideradas predadoras.

\section{Referências}

ALENCAR, J. A. de; HAJI, F. N. P.; BARBOSA, F. R.; Principais pragas do coqueiro e alternativas de controle. Petrolina, PE: Embrapa, 2002.

ARAGÃO, W. N.; CARVALHO, E. X. de; VASCONCELOS, K.; Caracterização morfológica reprodutiva de coqueiro-anão (Cocos nucifera $L$.) no litoral norte do Ceará. Aracaju, SE: Embrapa, 1998, 4p

ARAGÃO, W. M. Melhoramento genético do coqueiro. Aracaju, SE: Embrapa - CPATC, 1997. (EMBRAPA. Programa 07 - Matérias-primas. Subprojeto 07094020-03) Relatório.

GALlO, D.; NAKANO O.; SILVEIRA NETO S.; CARVALHO R. P. L.; BAPTISTA G. C.; FILHO E. B.; PARRA J. R. P.; ZUCCHI R. A.; ALVES S. B.; VENDRAMIN J. D.; MARCHINI L. C.; LOPES J. R. S.; OMOTO C. Entomologia agrícola. Piracicaba, SP: Fundação de Estudos Agrarios Luiz de Queiroz - FEALQ, 2002. 920 p.

GRAVENA, S. Insetos benéficos na Gravena. Disponível em: <http://www.gravena.com.br/insebenefico> Acesso em: 10 out. 2017.

GULLAN P. J.; CRANSTON, P. S. Insetos: Fundamentos da Entomologia. $5^{\text {a }}$ ed. Rio de Janeiro, 2017.

LEMOS W. P.; LINDS P. M. P.; TREVISAN O. Palmeiras. Em: SILVA N. M.; ADAIME R.; ZUCCHI R. A. (Editores técnicos). Pragas agrícolas e florestais na Amazônia. $1^{\text {a }}$ Ed. Brasília, DF: Embrapa, 2016. 608 p.

LIXA, A. T. Coccinellidae (Coleoptera) usando plntas aromáticas como Sítios de Sobrevivencia e Reprodução em sistema Agroecológico, e Aspectos Biológicos em condições de Laboratório. 2008. 77p. Dissertação (Mestrado em Fitossanidade e Biotecnologia aplicada). Instituto de biologia, Departamento de entomologia e fitopatologia. Universidade Federal Rural do Rio de Janeiro, Seropédica, RJ, 2008.

SEMA. Classificação Climática do Pará Método de Köppen. 2017. Disponível em: <www.sema.pa.gov.br/download/classificacao_climatica_do_para.doc>.Acesso em 10 out. 2017.

SILVEIRA NETO S.; NAKANO O.; BARBIN D.; VILLA NOVA N.A.; Manual de ecologia dos insetos. Piracicaba: Ceres; 1976.

TRIPLEHORN, C. A. \& JOHNSON, N. F. Estudo dos insetos. Tradução da $7^{a}$ edição de Borror and Delong's introduction to the study of insects. 2a ed., São Paulo: Cengage Learning, 2015. 809 p. 